\title{
NOVEL IMMUNOHISTOCHEMICAL MARKERS FOR THE DIFFERENTIATION OF LOBULAR AND DUCTAL INVASIVE BREAST CARCINOMAS
}

\author{
Gulisa Turashvilia*, Jan Bouchal ${ }^{\mathrm{a}}$, Jiri Ehrmann", Eduard Fridman ${ }^{\mathrm{b}}$, Jozef Skarda ${ }^{\mathrm{a}}$, \\ Zdenek Kolar ${ }^{\mathrm{a}}$ \\ a Laboratory of Molecular Pathology, Department of Pathology, Faculty of Medicine and Dentistry, Palacky University, \\ Olomouc, Czech Republic \\ ${ }^{b}$ Department of Pathology, Tel Aviv University, Chaim Sheba Medical Center and Sackler School of Medicine, Tel Aviv, \\ Israel \\ e-mail:guliat@gmail.com
}

Received: March 22, 2007; Accepted: May 18, 2007

Key words: Breast cancer/Ductal carcinoma/Lobular carcinoma/Immunohistochemistry/E-cadherin/Tissue microarray

Aims. Invasive ductal and lobular carcinomas are the most common histological types of breast cancer. The loss of E-cadherin expression has been suggested to be the most reliable marker for invasive lobular carcinoma. The aim of our study was to identify the diagnostic usefulness of novel markers in the differentiation of these tumor types.

Methods. We examined tissue microarrays (TMA) which were constructed from surgical specimens of 119 breast cancer patients. TMA consisted of 80 ductal carcinomas, 29 lobular carcinomas and special type cancers. TMA sections were stained using standard immunohistochemical methods. Monoclonal mouse antibodies against E-cadherin, cytokeratin 5/6 and 17, and polyclonal mouse antibodies against EMP1, DDR1, PRKCI and DVL1 were used.

Results. E-cadherin was absent in $93.3 \%$ of lobular tumors compared with only $15 \%$ of ductal tumors $(p<0.0001)$. EMP1 and DVL1 were overexpressed in lobular tumors (93.1\% and 96.5\%, respectively), whereas PRKCI and DDR1 were positive in ductal cancers (90\% and $96.2 \%$, respectively). Reduced expression or absence of both cytokeratins $5 / 6$ and 17 was found in both tumor tissues in comparison to normal terminal duct lobular units $(p<0.0001)$.

Conclusions. Apart from the well-established marker, E-cadherin, proteins examined on TMA slides by immunohistochemistry (EMP1, DVL1, DDR1, PRKCI) may represent novel tissue markers helpful in the differentiation of ductal and lobular breast cancers. Further studies with larger sets of patients are desirable, to verify the complete immunohistochemical profiles of various histological types of breast cancer and determine the prognostic and predictive significance of novel markers.

\section{BACKGROUND}

Invasive ductal (IDC) and lobular carcinomas (ILC) are the most common histological types of breast cancer, accounting for $80 \%$ and $15 \%$ of all malignant breast tumors, respectively. Microscopically, ductal carcinomas tend to form glandular structures, whereas lobular tumors are less cohesive and invade in single files ${ }^{23,33}$. Both tumor types derive from the breast terminal duct lobular units (TDLUs) ${ }^{28}$. The terminology "ductal" and "lobular" is still being used for historical reasons and to date there is no evidence to suggest that these tumors arise from ductal or lobular epithelial cells. Thus the differences in their morphology are likely to reflect the differences in mechanisms of carcinogenesis rather than the anatomical origin of the lesions.

IDC and ILC are similar in many respects ${ }^{1,8}$. However, clinical follow-up data and the patterns of metastasis suggest that their development and progression are different $^{26,31}$. The treatment for stage-matched tumors is similar ${ }^{18}$ but lobular carcinomas are often resistant to neoadjuvant therapy ${ }^{21}$. Although patients with ILCs are older, have low grade tumors and less lymphatic vascular invasion, they have no survival advantage compared with IDCs ${ }^{16}$. It is well documented that inactivating mutations and loss of heterozygosity of the CDH1 (E-cadherin gene), mapped to chromosome $16 \mathrm{q} 22.1$, are very frequent in $\mathrm{ILC}^{2,24}$, followed by the loss of protein expression ${ }^{12,15}$. E-cadherin is a calcium-dependent, epithelial-specific cell-cell adhesion molecule ${ }^{10}$. Simultaneous loss of E-cadherin and associated proteins such as the $\alpha$-, $\beta$ - and $\gamma$-catenins is an important step in the formation of lobular in situ carcinoma, a precursor of $\mathrm{ILC}^{20}$. Abnormal cytoplasmic and nuclear localization of p120, a member of the E-cadherin/catenin adhesion complex, occurs in the early stages of ILC and is maintained during tumor progression and metastasis. Most lobular carcinomas show cytoplasmic localization of p120 which is strongly associated with complete loss of E-cadherin and $\beta$-catenin. In ductal tumors, in contrast, reduction of p120 and E-cadherin in the membrane is common, whereas cytoplasmic p120 staining is rarely seen ${ }^{25}$. Maspin is a recently described member of the serpin family of protease inhibitors known to be a tumor suppressor gene product. It is more frequently detected in IDC than in ILC $(36.4 \%$ versus $7.1 \%)\left(\right.$ ref. $\left.^{14}\right)$. The expression of NKX3.1, a member of the NK-class of homeodomain, 
Table 1. Clinical and histopathological characteristics of TMA cases.

\begin{tabular}{|c|c|c|c|c|c|c|c|c|c|c|c|}
\hline \multicolumn{2}{|c|}{ Tumor } & G1 & $\mathrm{G} 2$ & G3 & ER+ & PgR+ & HER-2+ & Bcl-2+ & $\mathrm{N}_{0}$ & $\mathrm{~N}_{1}$ & $\mathrm{~N}_{2}$ \\
\hline \multirow{2}{*}{$\begin{array}{c}\text { IDC } \\
(\mathbf{n}=\mathbf{8 0})\end{array}$} & No & 6 & 57 & 17 & 57 & 50 & 12 & 13 & 56 & 16 & 8 \\
\hline & $\%$ & 7.5 & 71.3 & 21.2 & 71.3 & 62.5 & 15 & 16.3 & 70 & 20 & 10 \\
\hline \multirow{2}{*}{$\begin{array}{c}\text { ILC } \\
(n=29)\end{array}$} & No & 15 & 11 & 3 & 19 & 17 & 1 & 4 & 21 & 6 & 2 \\
\hline & $\%$ & 51.7 & 37.9 & 10.3 & 65.5 & 58.6 & 3.4 & 13.8 & 72.4 & 20.7 & 6.9 \\
\hline
\end{tabular}

ER, estrogen receptor; G1-3, grade 1-3; IDC, invasive ductal carcinoma; ILC, invasive lobular carcinoma; $\mathrm{N}_{0-2}$, lymph node status; PgR, progesterone receptor; TMA, tissue microarray.

Table 2. Results of immunohistochemical staining.

\begin{tabular}{|c|c|c|c|c|c|}
\hline \multirow{2}{*}{ Antibodies } & \multicolumn{2}{|c|}{ IDC (n= 80) } & \multicolumn{2}{c|}{ ILC (n = 29) } & \multirow{2}{*}{$\begin{array}{c}\text { Fisher's } \\
\text { P value }\end{array}$} \\
\cline { 2 - 5 } & No & \% & No & $\%$ & $<0.0001$ \\
\hline E-cadherin & 68 & 85 & 2 & 6.7 & $<0.0001$ \\
\hline DVL1 & 20 & 25 & 28 & 96.5 & $<0.0001$ \\
\hline DDR1 & 77 & 96.2 & 4 & 13.8 & $<0.0001$ \\
\hline EMP1 & 13 & 16.3 & 27 & 93.1 & $<0.0001$ \\
\hline PRKCI & 72 & 90 & 4 & 13.8 & \\
\hline
\end{tabular}

DVL1, human homolog of the Drosophila dishevelled gene; DDR1, discoidin domain receptor 1; EMP1, epithelial membrane protein 1; IDC, invasive ductal carcinoma; ILC, invasive lobular carcinoma; PRKCI, protein kinase C, iota.

is also found primarily in ILC ${ }^{9}$. To date, however, E-cadherin is the only well-established immunohistochemical marker for the differentiation of ductal and lobular breast carcinomas.

\section{METHODS}

To assess the diagnostic usefulness of novel markers identified by microarray analysis, we examined tissue microarrays (TMA) which were constructed from surgical specimens of 119 breast cancer patients aged >35 years and contained 278 cores of $2.0 \mathrm{~mm}$ diameter. They consisted of 80 ductal carcinomas, 29 lobular carcinomas, one tubular carcinoma, 3 medullary carcinomas, 2 tubular-lobular carcinomas, 2 mixed ductal-lobular carcinomas, one mucinous and one papillary carcinoma. Invasive ductal no special type (NST) tumors and invasive lobular carcinomas were graded using the Nottingham combined histologic grading system. The construction of the tissue microarrays was done using a tissue arrayer (Beecher Instruments, Inc., Sun Prairie, WI, USA)(ref. ${ }^{27}$ ).
Paraffin sections from 22 additional breast cancer samples containing normal mammary gland structures were used for comparison.

TMA sections were stained using standard immunohistochemical methods. Used were monoclonal mouse antibodies against E-cadherin (dilution 1:50, clone $\mathrm{NCH}-$ 38, Dako), cytokeratin 5/6 (dilution 1:100, clone D5/16 B4, Dako, Denmark) and cytokeratin 17 (dilution 1:100, clone 2D4-1G9, Abnova, Taipei, Taiwan), polyclonal mouse antibodies against EMP1 (epithelial membrane antigen 1; dilution 1:100, Abnova), DDR1 (discoidin domain receptor 1; dilution 1:100, Abnova), PRKCI (protein kinase C, iota, dilution 1:100, Abnova), and DVL1 (human homolog of the Drosophila dishevelled gene; dilution 1:100, Abnova). Microwave antigen retrieval was performed in citrate buffer ( $\mathrm{pH}$ 6.0). For antigen visualization, the EnVision/HRP system and DAB+ (Dako) were used. Slides were subsequently counterstained with hematoxylin, dehydrated and mounted in Canadian balsam. Immunohistochemical procedure was optimized by testing different antigen retrieval methods and using negative controls. 

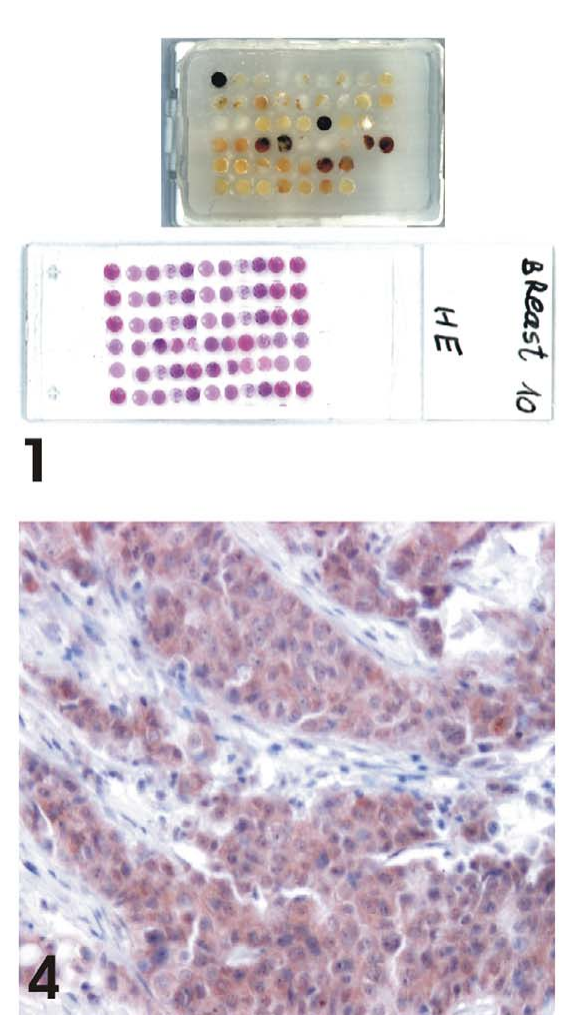
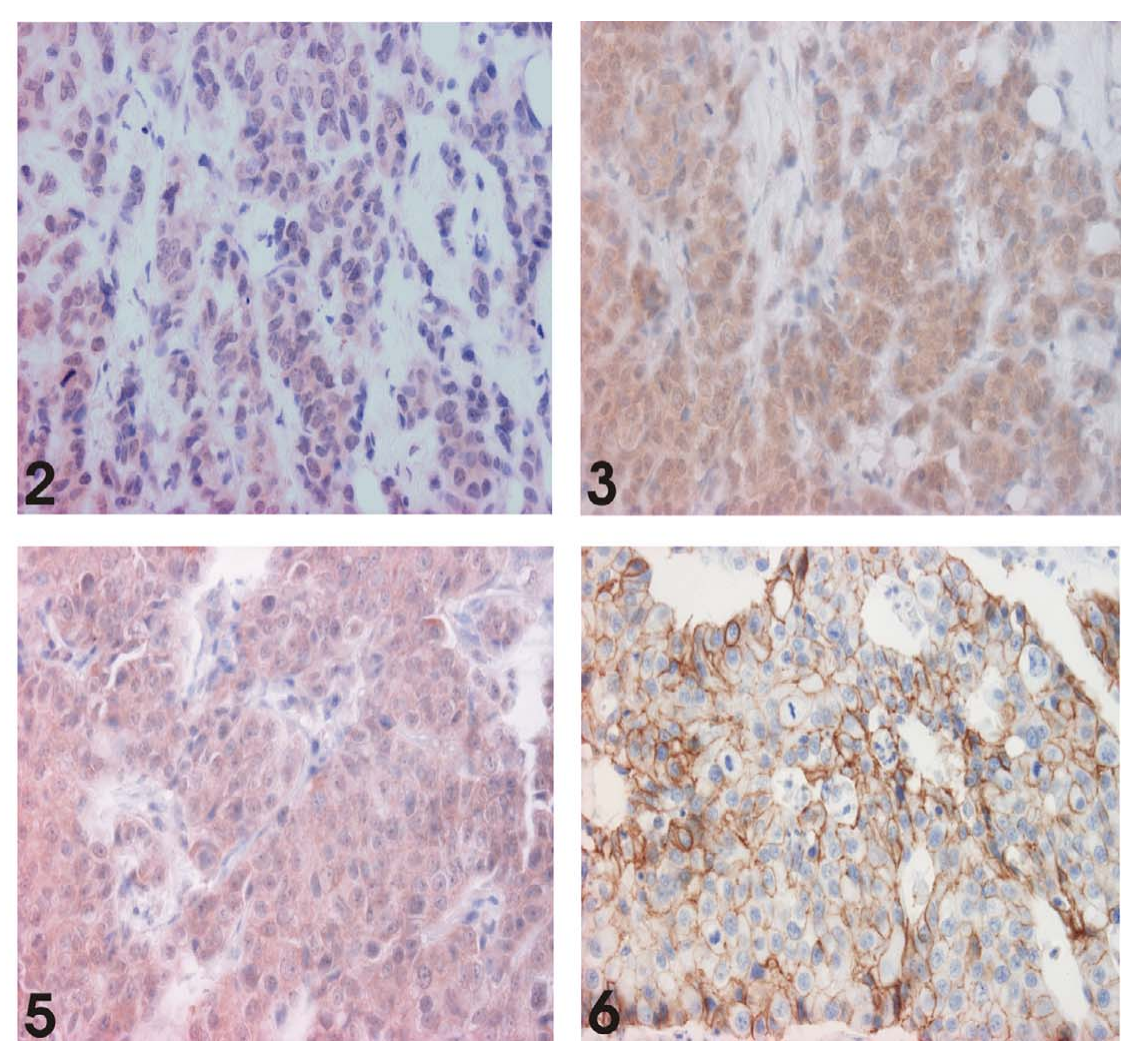

Fig. 1. Representative immunohistochemical staining for E-cadherin, DDR1, DVL1, PRKCI and EMP1. 1. TMA block and slide. 2. EMP1 is positive in lobular carcinoma; 3. DVL1 is positive in lobular carcinoma. 4. PRKCI is positive in ductal carcinoma. 5. DDR1 is positive in ductal carcinoma. 6. E-cadherin is positive in ductal carcinoma.

We regarded cells as immunoreactive when an obvious membranous or submembranous (E-cadherin, DDR1, EMP1) and/or cytoplasmic (cytokeratins, DVL1) staining was seen. Immunoreactivity was scored as follows: retained $(++)$ when more than $50 \%$ of membrane/cytoplasm was strongly positive, reduced $(+)$ when $10-50 \%$ of the membrane/cytoplasm was positive, and absent (-) when $0-10 \%$ of the membrane/cytoplasm was positive. Frequencies of positive and negative staining in tumor and normal tissues were compared using Fisher's exact test (http://home.clara.net/sisa/fisher.htm). The number of positive and negative staining results were combined for each antibody and the diagnostic sensitivity and specificity calculated using standard statistical methods ${ }^{3}$.

\section{RESULTS}

Of 80 invasive ductal breast carcinomas, $7.5 \%$ were grade $1,71.3 \%$ were grade 2 and $21.2 \%$ were grade 3 . Of 29 invasive lobular carcinomas, $51.7 \%$ were grade 1 , $37.9 \%$ were grade 2 and $10.3 \%$ were grade 3 . Lymph node status was classified as $\mathrm{N}_{0}$ in $70 \%$ of invasive ductal carcinomas and $72.4 \%$ of invasive lobular carcinomas, $\mathrm{N}_{1}$ in $20 \%$ of invasive ductal carcinomas and $20.7 \%$ of invasive lobular carcinomas, $\mathrm{N}_{2}$ in $10 \%$ of invasive ductal carcinomas and $6.9 \%$ in invasive lobular carcinomas. According

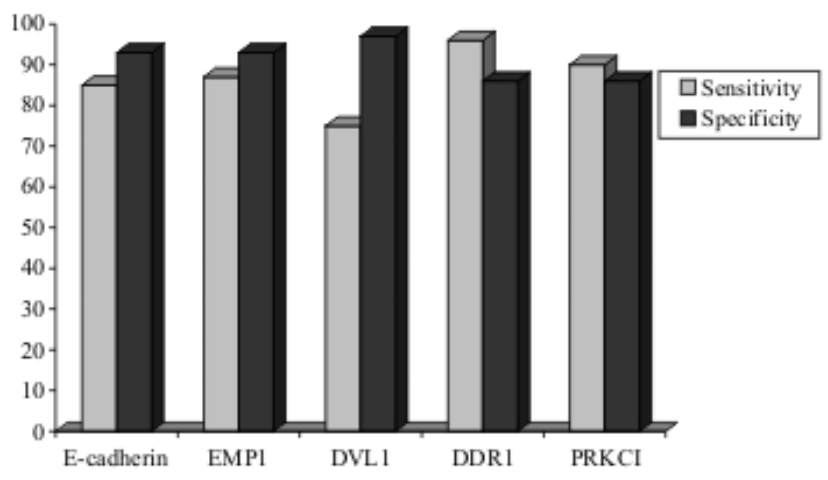

Fig. 2. Diagnostic sensitivity and specificity of immunohistochemical markers for distinguishing invasive ductal and lobular breast carcinomas.

to routine immunohistochemical data, $71.3 \%$ of invasive ductal carcinomas were positive for estrogen receptors (ER), and $62.5 \%$ were positive for progesterone receptors (PR), whereas $65.5 \%$ of lobular carcinomas were ER-positive, and $58.6 \%$ were PR-positive. Immunohistochemical protein overexpression and subsequent amplification of c-erbB-2 (HER-2/neu) gene by fluorescent in situ hybridization (FISH) was detected in $15 \%$ of invasive ductal carcinomas and $3.4 \%$ of invasive lobular carcinomas. The clinical and histopathological characteristics of these patients are shown in Table 1. 

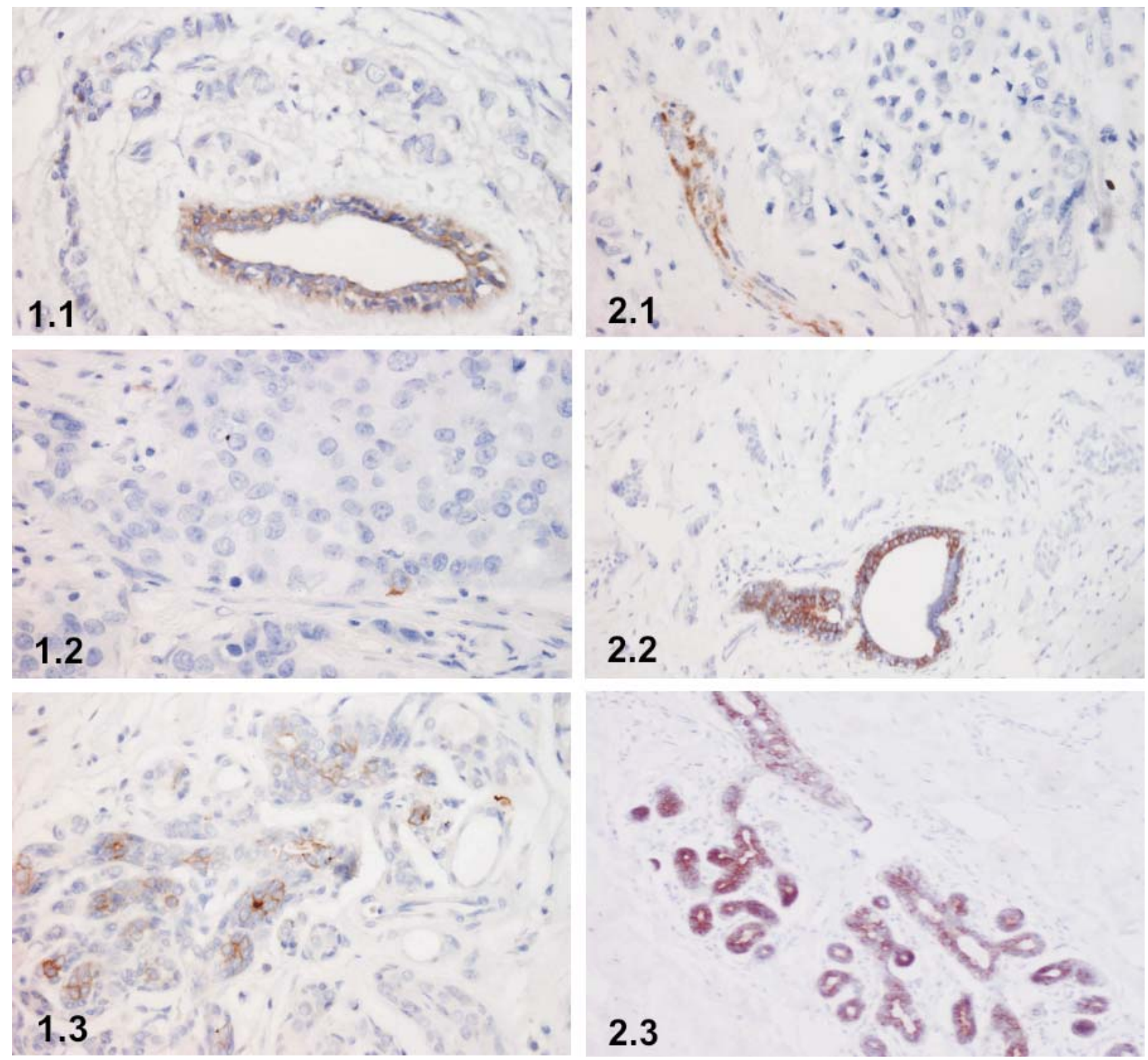

Fig. 3. Representative immunohistochemical staining for cytokeratins. 1.1. Cytokeratin $5 / 6$ is negative in lobular carcinoma cells, but its expression is retained in normal ductal epithelial cells; 1.2 . Cytokeratin $5 / 6$ is negative in ductal carcinoma cells; 1.3. Duct lobular unit in normal mammary gland tissue is positive for cytokeratin $5 / 6$; 2.1. Cytokeratin 17 is negative in lobular carcinoma cells, but its expression is retained in normal ductal epithelial cells; 2.2. Cytokeratin 17 is negative in ductal carcinoma cells, but its expression is retained in normal ductal epithelial cells; 2.3. Duct lobular unit in normal mammary gland tissue is positive for cytokeratin 17.

E-cadherin successfully distinguished invasive ductal and lobular carcinomas. It was absent in $93.3 \%$ of lobular tumors compared with only $15 \%$ of ductal tumors $(p<0.0001)$. EMP1, PRKCI, DDR1 and DVL1 were also differentially expressed between ductal and lobular carcinomas. More precisely, $93.1 \%$ of lobular tumors were positive for EMP1 compared to only $16.3 \%$ of ductal tumors $(\mathrm{p}<0.001)$. Ductal carcinomas were positive for PRKCI in $90 \%$ of cases compared with only $13.8 \%$ of lobular carcinoma ( $p<0.001$ ). DDR1 was positive in 96.2 $\%$ of ductal carcinomas compared to only $13.8 \%$ of lobular carcinoma ( $p<0.001)$ (Fig. 1). Also, $96.5 \%$ of lobular tumors were positive for DVL1 compared with only $25 \%$ of ductal tumors $(p<0.001)$ (Tab. 2). Thus, the diagnostic sensitivity and specificity of E-cadherin for distinguishing IDC and ILC were $85 \%$ and $93 \%$, respectively. The sensitivity and specificity were $87 \%$ and $93 \%$ for EMP1, $75 \%$ and $97 \%$ for DVL1, $96 \%$ and $86 \%$ for DDR 1 , and $90 \%$ and $86 \%$ for PRKCI (Fig. 2).

The reduced expression or absence of both cytokeratins 5/6 and 17 was found in both tumor tissues in comparison to terminal duct lobular units in 22 normal mammary tissues $(p<0.0001)$ (Fig. 3). In a majority of ducts and lobules including TDLU, these cytokeratins were expressed in both basal and luminal cells. The expression of these cytokeratins was similar in IDC and ILC. Cytokeratin 5/6 
was positive in $11.2 \%$ of invasive ductal carcinomas and $10.3 \%$ of invasive lobular carcinomas, and cytokeratin 17 was expressed in $10 \%$ and $6.9 \%$, respectively.

Of the special type carcinomas included on TMA slides, a papillary and two medullary carcinomas were positive for cytokeratin $5 / 6$, one out of three medullary carcinomas was positive for EMP1 and E-cadherin, and a ductal-lobular carcinoma was positive for DDR1. All other special type carcinomas were negative for these markers, and finally none were positive for either PRKCI or DVL1 (data not shown).

\section{DISCUSSION}

We studied the expression of novel immunohistochemical markers in 119 surgical samples from patients with lobular and ductal invasive breast carcinomas. The results of immunostaining suggest that two tumor types can be successfully distinguished at the protein level. Most importantly, E-cadherin was negative in $92.3 \%$ of lobular carcinomas. Its diagnostic sensitivity and specificity for distinguishing IDC and ILC were $85 \%$ and $93 \%$, respectively. The gene encoding E-cadherin protein acts as a tumor suppressor, inhibiting invasion and metastasis. Its mutations correlate with gastric, breast, colorectal, thyroid and ovarian cancer. During tumor progression, E-cadherin can be functionally inactivated or silenced by different mechanisms such as post-translational control, somatic mutations, downregulation of gene expression through promoter hypermethylation, histone deacetylation, and, importantly, transcriptional repression ${ }^{11,22}$. The latter induces cellular responses leading to the conversion of epithelial cells into invasive mesenchymal-like cells with increased motility and invasiveness. This process is called an epithelial-mesenchymal transition (EMT) $\left(\right.$ ref. $\left.^{22}\right)$. The loss of E-cadherin expression explains the morphological characteristics of invasive lobular carcinomas such as Indian file arrangement of tumor cells.

In addition to E-cadherin, other proteins involved in cell adhesion and proliferation are differentially expressed between ductal and lobular carcinomas. PRKCI is a calcium-independent, phospholipid-dependent, serine- and threonine-specific enzyme which may play a role in the secretory response to nutrients. It is involved in cell polarization processes and the formation of epithelial tight junctions ${ }^{4}$. PRKCI was overexpressed in our invasive ductal carcinomas, and its diagnostic sensitivity and specificity for distinguishing IDC and ILC were $90 \%$ and $86 \%$, respectively. EMP1, a tumor-associated membrane protein involved in cell-cell interactions and control of cell proliferation ${ }^{7,35}$, was overexpressed in lobular carcinomas, and its diagnostic sensitivity and specificity for distinguishing IDC and ILC were $87 \%$ and $93 \%$, respectively.

DDR1 protein was overexpressed in ductal tumors and this agrees with the literature ${ }^{5}$. The diagnostic sensitivity and specificity of DDR1 for distinguishing IDC and ILC were $87 \%$ and $93 \%$, respectively. Expression of this receptor tyrosine kinase is restricted to epithelial cells and determines the regulation of cell growth, differentiation and metabolism ${ }^{6}$. In addition, G-protein signaling may mediate the effect of Wnt5a expression by enabling collagen-induced activation of DDR $1^{6}$, and binding DDR 1 to Wnt-5 regulates the adhesion of mammary cells ${ }^{5-6,13}$. The Wnt proteins constitute a large family of secreted signaling molecules that are implicated in the early mesodermal patterning of the embryo, in regulation of mammary gland ductal branching and alveolar morphogenesis during pregnancy, also in tumorigenesis ${ }^{17,29}$. Wnt signal transduction starts via binding to cell surface Frizzled receptors. Further signaling depends on $\beta$-catenin activation (canonical pathway) or involves intracellular molecules other than $\beta$-catenin (non-canonical pathway) $\left(\right.$ ref. $^{34}$ ). It is well documented that DVL1 is an essential mediator of both canonical and non-canonical Wnt pathways ${ }^{34}$. Amplification and increased expression of the DVL1 gene has previously been associated with breast carcinogenesis $^{19}$. However, histological subtypes have not been specified. Of Wnt molecules, DVL1 was overexpressed in our invasive lobular carcinomas, and its diagnostic sensitivity and specificity for distinguishing IDC and ILC were $75 \%$ and $97 \%$, respectively.

In a majority of ducts and lobules including TDLU in normal mammary tissues, cytokeratins $5 / 6$ and 17 were expressed in both basal and luminal cells, confirming the previously described variability of expression of these basal cytokeratins and their relationship to cellular origin ${ }^{30}$. Cytokeratin 5 and 17 have also been found in a subset of breast cancer patients with a poor clinical outcome ${ }^{32}$. In our set of patients, the expression of these basal cytokeratins was associated with higher tumor grade and HER-2/ neu-negativity $(p<0.001)$. No other immunohistochemical markers correlated with clinical or histopathological markers $(p>0.05)$.

\section{CONCLUSION}

Taken together, our results suggest that besides the well-established marker E-cadherin, proteins examined on TMA slides by immunohistochemistry (EMP1, DVL1, DDR1, PRKCI) may represent novel tissue markers helpful in the differentiation of ductal and lobular breast cancers. Further studies with larger sets of patients are desirable to verify the complete immunohistochemical profiles of various histological types of breast cancer and determine prognostic and predictive significance of novel markers.

\section{ACKNOWLEDGEMENTS}

The work was supported by grants NR 7844-3 and NR 8425-3 from the Ministry of Health and MSM 6198959216 from the Ministry of Education, Youth and Sports of the Czech Republic. 


\section{REFERENCES}

1. Arpino G, Bardou VJ, Clark GM, Elledge RM. Infiltrating lobular carcinoma of the breast: tumor characteristics and clinical outcome. Breast Cancer Res 2004;6:R149-56.

2. Asgeirsson KS. Altered expression of E-cadherin in breast cancer, patterns, mechanisms and clinical significance. Eur J Cancer 2000;36:1098-106.

3. Daly LE, Bourke GJ. Interpretation and uses of medical statistics 5th edn. Oxford: Blackwell Science 2000; 412-5.

4. De Donato M, Gallagher Jr. DS, Davis SK, Stelly DM, Taylor JF. The assignment of PRKCI to bovine chromosome 1q34-q36 by FISH suggests a new assignment to human chromosome 3 . Cytogenet. Cell Genet. 2001;95:79-81.

5. Dejmek J, Dib K, Jonsson M, Andersson T. Wnt-5a and G-protein signaling are required for collagen-induced DDR 1 receptor activation and normal mammary cell adhesion. Int $\mathrm{J}$ Cancer 2003; 103:344-51.

6. Dejmek J, Leandersson K, Manjer J, Bjartell A, Emdin SO, Voge $\mathrm{WF}$, et al. Expression and signaling activity of Wnt-5a/discoidin domain receptor-1 and Syk plays distinct but decisive roles in breast cancer patient survival. Clin Cancer Res 2005;11:520-8.

7. Durmus T, Leclair RJ, Park KS, Terzic A, Yoon JK, Lindner V. Expression analysis of the novel gene collagen triple helix repeat containing-1 (Cthrc1). 2006;6:935-40

8. Ferlicot S, Vincent-Salomon A, Medioni J, Genin P, Rosty C, SigalZafrani B, et al. Wide metastatic spreading in infiltrating lobular carcinoma of the breast. Eur J Cancer 2004;40:336-41.

9. Gelmann EP, Bowen C, Bubendorf L. Expression of NKX3.1 in normal and malignant tissues. Prostate 2003;55:111-7.

10. Goldstein NS, Bassi D, Watts JC, Layfield LJ, Yaziji H, Gown AM. E-cadherin reactivity of 95 noninvasive ductal and lobular lesions of the breast. Implications for the interpretation of problematic lesions. Am J Clin Pathol 2001;115:534-42.

11. Grille SJ, Bellacosa A, Upson J, Klein-Szanto AJ, van Roy F, LeeKwon W, et al. The protein kinase Akt induces epithelial mesenchymal transition and promotes enhanced motility and invasiveness of squamous cell carcinoma lines. Cancer Res 2003;63:2172-8.

12. Gupta D, Croitoru CM, Ayala AG, Sahin AA, Middleton LP. Ecadherin immunohistochemical analysis of histiocytoid carcinoma of the breast. Ann Diagn Pathol 2002;6:141-7.

13. Jonsson M, Andersson T. Repression of Wnt-5a impairs DDR1 phosphorylation and modifies adhesion and migration of mammary cells. J Cell Sci 2001;114:2043-53.

14. Kim DH, Yoon DS, Dooley WC, Nam ES, Ryu JW, Jung KC, et al. Association of maspin expression with the high histological grade and lymphocyte-rich stroma in early-stage breast cancer. Histopathology 2003;42:37-42.

15. Kowalski PJ, Rubin MA, Kleer CG. E-cadherin expression in primary carcinomas of the breast and its distant metastases. Breast Cancer Res 2003;5:R217-22.

16. Mersin H, Yildirim E, Gulben K, Berberoglu U. Is invasive lobular carcinoma different from invasive ductal carcinoma? Eur J Surg Oncol 2003;29:390-5.

17. Miller JR. The Wnts. Genome Biol 2002;3:3001.1-3001.15.

18. Molland JG, Donnellan M, Janu NC, Carmalt HL, Kennedy CW, Gillett DJ. Infiltrating lobular carcinoma - a comparison of diag- nosis, management and outcome with infiltrating duct carcinoma. Breast 2004;13:389-96.

19. Nagahata T, Shimada T, Harada A, Nagai H, Onda M, Yokoyama $\mathrm{S}$, et al. Amplification, up-regulation and over-expression of DVL1 , the human counterpart of the Drosophila disheveled gene, in primary breast cancers. Cancer Sci 2003;94:515-8.

20. Nakopoulou L, Gakiopoulou-Givalou H, Karayiannakis AJ, Giannopoulou I, Keramopoulos A, Davaris P, Pignatelli M. Abnormal alpha-catenin expression in invasive breast cancer correlates with poor patient survival. Histopathology 2002;40:536-46.

21. Newman LA, Buzdar AU, Singletary SE, Kuerer HM, Buchholz T, Ames FC, et al. A prospective trial of preoperative chemotherapy in resectable breast cancer: predictors of breast-conservation therapy feasibility. Ann Surg Oncol 2002;9:228-34.

22. Peinado H, Portillo F, Cano A. Transcriptional regulation of cadherins during development and carcinogenesis. Int J Dev Biol 2004;48:365-375.

23. Rosen PP. Rosen's breast pathology. Lippincots-Raven Publishers; 1997.

24. Roylance R, Droufakou S, Gorman P, Gillett C, Hart IR, Hanby A, Tomlinson I: The role of E-cadherin in low-grade ductal breast tumorigenesis. Pathol 2003;200:53-8.

25. Sarrio D, Perez-Mies B, Hardisson D, Moreno-Bueno G, Suarez A, Cano A, et al. Cytoplasmic localization of p120ctn and E-cadherin loss characterize lobular breast carcinoma from preinvasive to metastatic lesions. Oncogene 2004;23:3272-83.

26. Silverstein MJ, Lewinsky BS, Waisman JR, Gierson ED, Colburn WJ, Senofsky GM, Gamagami P. Infiltrating lobular carcinoma. Is it different from infiltrating duct carcinoma? Cancer 1994;73:16737.

27. Simon R, Sauter G. Tissue microarrays for miniaturized high-throughput molecular profiling of tumors. Exp Hematol 2002;30:1365-72.

28. Simpson P, Reis-Filho J, Gale T, Lakhani S. Molecular evolution of breast cancer. J Pathol 2005;205:248-254.

29. Smalley M, Dale TC. Wnt signaling and mammary tumorigenesis. J. Mamm. Gland Biol. Neoplasia 2001;6:37-52.

30. Tlsty TD, Crawford YG, Holst CR, Fordyce CA, Zhang J, McDermott K, et al. Genetic and epigenetic changes in mammary epithelial cells may mimic early events in carcinogenesis. J Mamm Gland Biol Neoplasia 2004;9:263-74.

31. Toikkanen S, Pylkkanen L, Joensuu H. Invasive lobular carcinoma of the breast has better short- and long-term survival than invasive ductal carcinoma. Br J Cancer 1997;76:1234-40.

32. van de Rijn M, Perou CM, Tibshirani R, Haas P, Kallioniemi O, Kononen J, et al. Expression of cytokeratins 17 and 5 identifies a group of breast carcinomas with poor clinical outcome. Am J Pathol 2002;161:1991-6.

33. Weidner N, Cote R, Suster S, Weiss L. Modern Surgical Pathology. Elsevier Science; 2003.

34. Yamanaka H, Moriguchi T, Masuyama N, Kusakabe M, Hanafusa $\mathrm{H}$, Takada $\mathrm{R}$, et al. JNK functions in the noncanonical Wnt pathway to regulate convergent extension movements in vertebrates. EMBO Rep 2002;3:69-75.

35. Zhao X, Ramsey KE, Stephan DA, Russell P. Gene and protein expression changes in human trabecular meshwork cells treated with transforming growth factor-beta. Invest Ophthalmol Vis Sci 2004;45:4023-34. 